\title{
Mainlobe Jamming Suppression Using Improved Frequency Diverse Array with MIMO Radar
}

\author{
Guang-ming Li, ${ }^{1,2}$ Qun Zhang $\mathbb{D}^{1,2,3}$ Qi-yong Liu, ${ }^{4}$ Jia Liang, ${ }^{1,2}$ Dan Wang, ${ }^{1,2}$ \\ and Feng $\mathrm{Zhu}^{5,6}$ \\ ${ }^{1}$ Information and Navigation College, Air Force Engineering University, Xi'an 710077, China \\ ${ }^{2}$ Collaborative Innovation Center of Information Sensing and Understanding, Xi'an 710077, China \\ ${ }^{3}$ Key Laboratory for Information Science of Electromagnetic Waves, Fudan University, Shanghai 200433, China \\ ${ }^{4} 93116$ Unit of PLA, Shenyang 110000, China \\ ${ }^{5}$ National Defense University of PLA, Beijing 100091, China \\ ${ }^{6}$ Academy of Military Sciences of PLA, 100091, China \\ Correspondence should be addressed to Qun Zhang; zhangqunnus@gmail.com
}

Received 26 October 2018; Accepted 19 March 2019; Published 2 May 2019

Academic Editor: Antonio Lazaro

Copyright (C) 2019 Guang-ming Li et al. This is an open access article distributed under the Creative Commons Attribution License, which permits unrestricted use, distribution, and reproduction in any medium, provided the original work is properly cited.

Frequency diverse array (FDA) has attracted much attention in recent years due to its range-angle-dependent beampattern. Multiple-input multiple-output (MIMO) radar can offer waveform diversity to increase the virtual aperture length for azimuth coherent focus processing in radar imaging. Combining the advantages of FDA and MIMO radar, FDA-MIMO radar can steer multiple beams to different targets in the same line of sight (LOS) of radar with different waveforms. In this paper, an improved FDA model with the logistic map is proposed to get the aperiodic and range-angle uncoupling beampattern. Based on the proposed FDA, combining the FDA-MIMO radar, the waveform and chirp rate jitter techniques are adopted to mainlobe jamming suppression. Simulation results show the effectiveness of the proposed method.

\section{Introduction}

Due to the all-day, all-weather, long-range capability, the inverse synthetic aperture radar (ISAR) has played a critical role in space surveillance. In situations where other radars display only a single bright unidentifiable moving pixel, the ISAR image is adequate to discriminate between various missiles, military aircraft, and civilian aircraft. In order to protect the important goals and regions of detection and observation, a variety of jamming techniques are designed against the ISAR system. Sub-Nyquist sampling jamming against the ISAR system is studied in [1-4]. In [1], sub-Nyquist jamming is used to the bistatic ISAR; by undersampling the intercepted signal of ISAR, multiple deceptive false targets will be induced and the real targets will be emerged in false targets. Although ISAR jamming has got a significant progress, there are only rare studies about ISAR anti-jamming. In [5], the ISAR anti-jamming effect of planar array is analyzed, but this method can only eliminate the jamming signal from the sidelobe and is invalid for the mainlobe jamming (MLJ).

Currently, phased-array radar and multiple-input multiple-output (MIMO) radar are two common radar systems for target imaging [6]. Both phased-array radar and MIMO radar have their advantages and disadvantages, and phased-MIMO radar exploits the benefits of the phasedarray and MIMO radars [7]. There are multiple advantages of phased-MIMO radar, but the beampattern of phased-MIMO radar is just angle-dependent. In some electronic warfare scenarios, e.g., when the jammer and targets are in the same LOS of radar, ISAR will receive both the jamming signal and target echo. In this situation, the traditional electronic countercountermeasure (ECCM) method, such as a spatial filtering method, will degrade greatly because the jamming signal has a similar steering vector with the target echo. In order to detect 
targets from MLJ, many techniques have been proposed recently. MLJ suppression techniques on the monopulse sum-difference network have been studied in $[8,9]$. These methods can cancel MLJ by adaptive sum-difference channel cancellation, but restricted by the degree of freedom; and these methods just work when there is only one MLJ. Multiple MLJs can be cancelled by adaptive arrays in $[10,11]$, but the shape of the mainlobe would be distorted greatly and the mainlobe gain on target echoes will become very small. The block matrix preprocessing (BMP) and eigen-projection processing (EMP) methods can solve the mainlobe deformation, mainlobe shift, and sidelobe elevation problem $[12,13]$, but BMP has high accuracy requirement for the direction of jamming signal; otherwise, the effect of MLJ suppression will degrade greatly. EMP method does not need for accurate estimation for the direction of MLJ, but eigenvalue decomposition and inverse matrix operation increase the calculation cost seriously. There are many improved methods in recent years [14-16], but when MLJ is located close and even overlapped to the target, these techniques will be invalid. Considering the growing threats of MLJ, new techniques need to be studied.

In recent years, frequency diverse array (FDA), which features flexible beam scanning ability, has attracted growing attention. The FDA was first introduced by Antonik et al. in [17], where a minor carrier frequency offset is employed across the array elements. Different from phased array, FDA is capable of forming a range-angle-dependent beampattern, so FDA has the innate advantage in MLJ suppression. Due to the range-angle-coupled beampattern, basic FDA is not desirable for application. And the beampattern of FDA can be influenced by the frequency offset, so many frequency shift methods are studied to eliminate the range periodicity and decouple the range-angle-coupled response. In $[18,19]$, the frequency offset is controlled by logarithmic and sine law, respectively. The range-angle-coupled character has been overcome, but it has difficulties in forming high resolution beams.

Refer to phased-MIMO radar, FDA-MIMO radar has been proposed in [20-23]. The essence of FDA-MIMO radar is dividing the transmit array into some subarrays. Each subarray adopts the operating mode of FDA radar, which can get coherent processing in range-angle dimension with element level frequency shifting. And the signal emitted by each subarray is orthogonal or irrelevant. Compared with phased-MIMO radar, FDA-MIMO radar can transmit different signals in the same LOS of radar with different ranges, so it has the innate benefits in MLJ suppression. A subspace projection-based sample selection method is proposed in [24] to handle the pseudorandom distribution false targets, and the jammings can be suppressed adequately even if they are in the mainlobe direction. The key points of FDA-MIMO radar are the character of FDA and structure of FDA-MIMO radar, so in this paper, an improved FDA based on the logistic map is proposed to get the high-resolution range-angle-uncoupled beampattern. Then, the transmit array is divided into multiple subapertures according to the number of targets, and each subaperture operates in FDA mode. For each subaperture, the elements steer transmit beam in one of the targets' directions and ranges. To avoid echo signal separation of multitargets, it is guaranteed that there is only one target in each transmit beam by adjusting the subaperture dividing method. On the basis, orthogonal signals with different chirp rates and waveforms are transmitted to the jammer and targets, respectively. The jamming signal is mismatched with reference signal and will be eliminated.

Compared with MLJ suppression based on the phasedarray radar, the proposed method has three advantages: (1) according to the target and jamming characters, different waveforms will be transmitted to different targets even if they are in the same LOS of radar; (2) the time sampling can be replaced by spatial sampling; thus, the requirement of time resource can be decreased and the real-time performance can be increased; and (3) MLJ can be suppressed much easier compared with the complex null steering algorithm in phased array.

Compared with the anti-jamming method based on the MIMO radar, the proposed method has two advantages: (1) it has higher SNR gain, which leads to better antijamming effect; and (2) because the waveform emitted by each subaperture is orthogonal or irrelevant and there is only one target in each beam, the separated orthogonal signal obtained in the receiver will contain single target information; thus, the signal separation of multiple targets and jammers can be avoided.

The reminder of this paper is organized as follows. In Section 2, an improved FDA is proposed and the FDAMIMO based on the improved FDA is introduced; experiments are given to demonstrate effectiveness in Section 3. Finally, conclusion and following points are given in Section 4 .

\section{Model of Improved FDA-MIMO Radar}

2.1. Model of Improved FDA. Assuming the basic FDA radar is a uniform linear array formed byMtransmit antennas and defines the first element as reference. The transmit frequency of the $m$-th element is

$$
f_{m}=f_{0}+(m-1) \Delta f, \quad m=1,2, \ldots, M,
$$

where $f_{0}$ is the reference carrier frequency and $\Delta f$ is the basic frequency offset. In general, $(M-1) \Delta f \ll f_{0}$. For a target located at $(\theta, r)$ in the far field, neglecting the high-order phase term $[25,26]$, the transmit steering vector can be expressed as

$$
a(\theta, r)=\left[1, \cdots, \exp \left(-j \frac{2 \pi(M-1)}{c}\left(\Delta f r-f_{0} d \sin \theta\right)\right)\right]
$$

The array factor is

$$
\operatorname{AF}(\theta, r)=\sum_{m=1}^{M} \exp \left(-j \frac{2 \pi(m-1)}{c}\left(\Delta f r-f_{0} d \sin \theta\right)\right)
$$


With frequency offset as formula (1), the beam of basic FDA is shown in Figure 1 [17].

The application of basic FDA is limited by the periodicity and coupling, which result in ambiguously positioning targets in range-angle dimension. In [27], a new FDA structure is proposed, the nonuniform FDA is exploited as a transmitter to provide range-dependent transmitting beampattern, and the uniform phased array is exploited as a receiver to provide angle-dependent receiving beampattern. The key point of this FDA structure is the nonuniform FDA, but this needs high accuracy of space between array elements. Considering the cost and difficulties in achieving, we prefer to improve the beampattern by changing the frequency offset in two ways: (1) increase the frequency offset and (2) change the frequency shift method. Because the frequency offset cannot be too large, or the signal's phase emitted by FDA is incoherent among elements and cannot form the desired beam in the spatial domain, in this paper, we propose a new frequency offset shift method to achieve the range-angle decouple and high resolution of the beam.

The logistic map, one form of chaos theory, has attracted wide attention in recent decades. The sequence created by a logistic map has the similar distribution and probability character with the white noise [28]. In this paper, we try to make the FDA's frequency offset shifted with the logistic map, and the FDA proposed in this paper is called L-FDA. Figure 2 is the system sketch of L-FDA [29]. There are $M$ elements in a linear array, with a constant interelement spacing $d$. These array elements are located symmetrically along the $x$-axis; the original point of the $x$-axis is selected as the first element, and the coordinate of the $m$-th element is

$$
x_{m}=(m-1) d, \quad m=1,2, \ldots, M .
$$

The transmitted waveform of each element is the same, but the carrier frequencies of different elements are randomly assigned. The frequency offset of the $m$-th element is

$$
\Delta f_{m+1}=\mu \Delta f_{m}\left(1-\Delta f_{m}\right), \quad m=1,2, \ldots, M .
$$

$\mu \in[0,4]$ is the control parameters; when $\mu \in(3.5699456$ , 4], the logistic map is in the chaotic region. The transmitted waveform of $m$-th element can be expressed by

$$
s_{m}(t)=\exp \left(j 2 \pi\left(f_{0}+\Delta f_{m}\right) t\right)
$$

Define the first element as reference, with the far-field assumption for angle $\theta$ and range $r$, L-FDA is utilized as the transmit array; then, the signal that transmitted by the $m$-th element is

$$
\begin{aligned}
s r_{m}(t ; \theta, r)= & s_{m}\left(t-\frac{r-x_{m} \sin \theta}{c}\right) \\
= & \exp \left(j 2 \pi\left(f_{0}+\Delta f_{m}\right)\right) \\
& \cdot\left(t-\frac{r-x_{m} \sin \theta}{c}\right),
\end{aligned}
$$

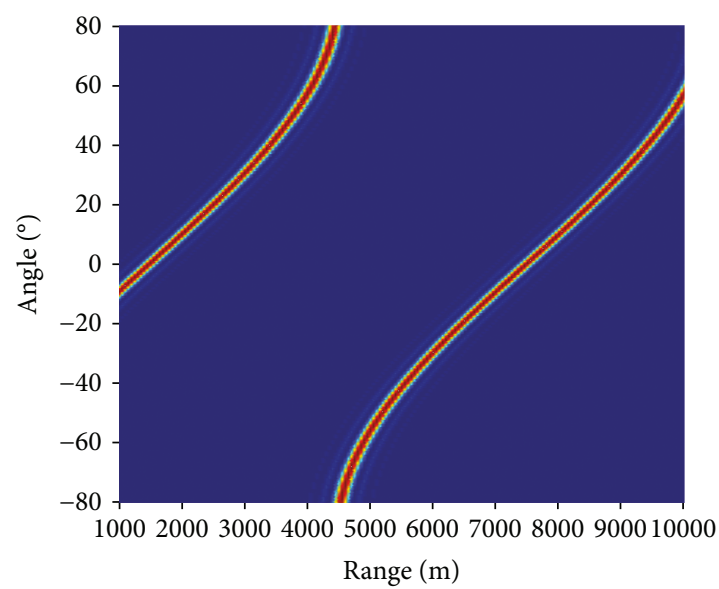

FIGURE 1: Beampattern of basic FDA.
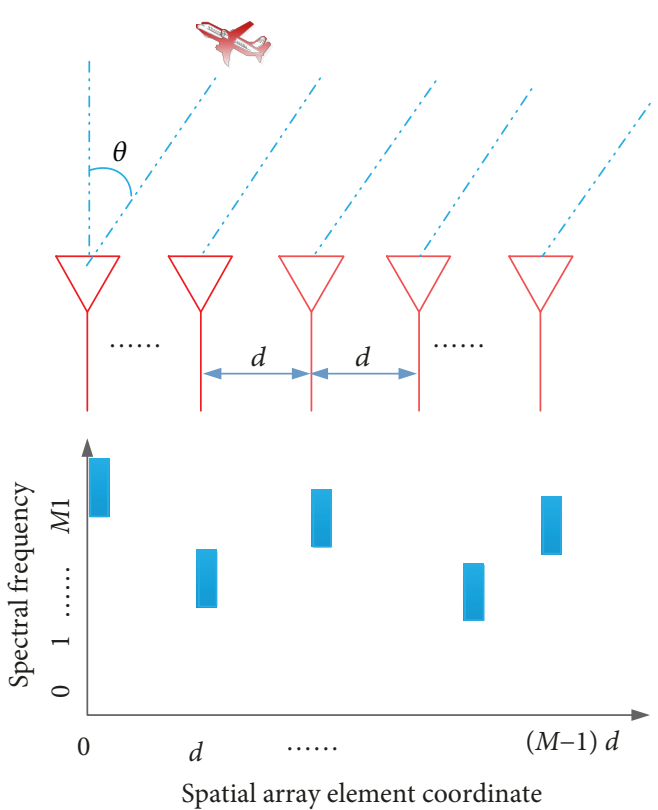

Figure 2: System sketch of L-FDA.

where $c$ is the wave propagation speed. The baseband signal that transmitted by the $m$-th element is

$$
\Psi_{m}(\theta, r)=\exp \left(-j \frac{2 \pi}{c}\left(f_{0}+\Delta f_{m}\right)(r-(m-1) d \sin \theta)\right)
$$

The baseband samples $\Psi_{m}(\theta, r)$ of all elements can be arranged to formulate a range-angle-dependent steering vector:

$$
\Psi(\theta, r)=\left[\Psi_{0}(\theta, r), \Psi_{1}(\theta, r), \cdots, \Psi_{M-1}(\theta, r)\right]=\Psi(\theta) \odot \Psi(r),
$$




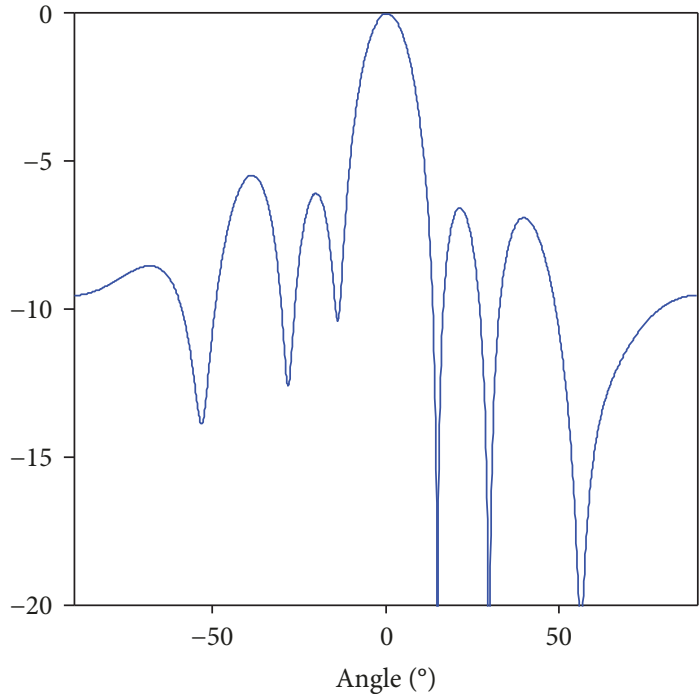

(a)

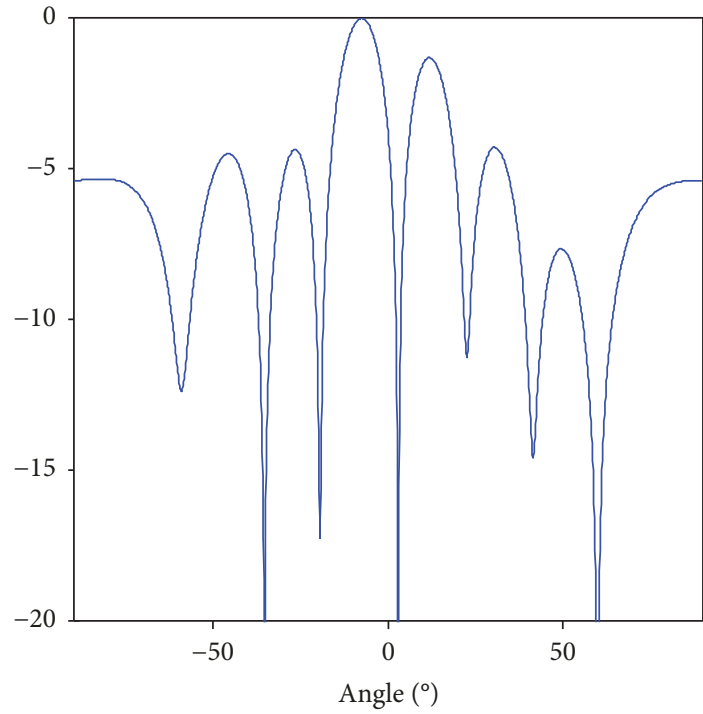

(c)

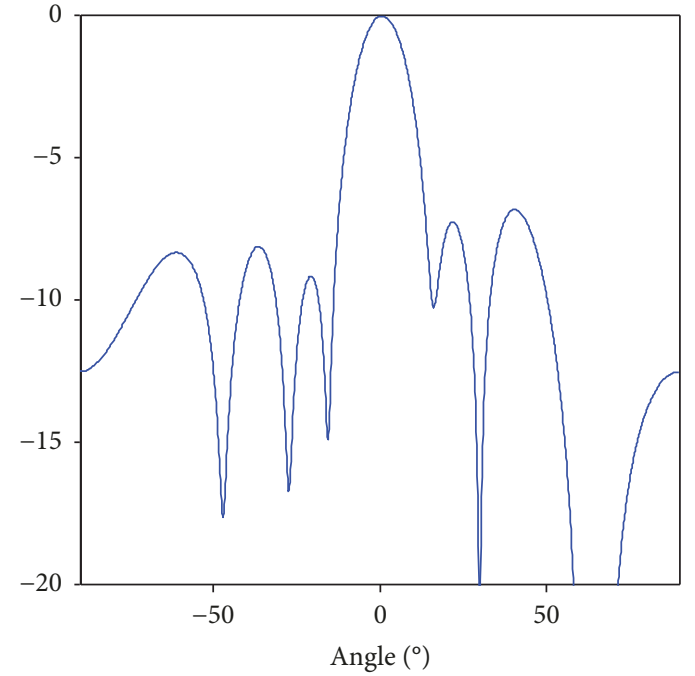

(b)

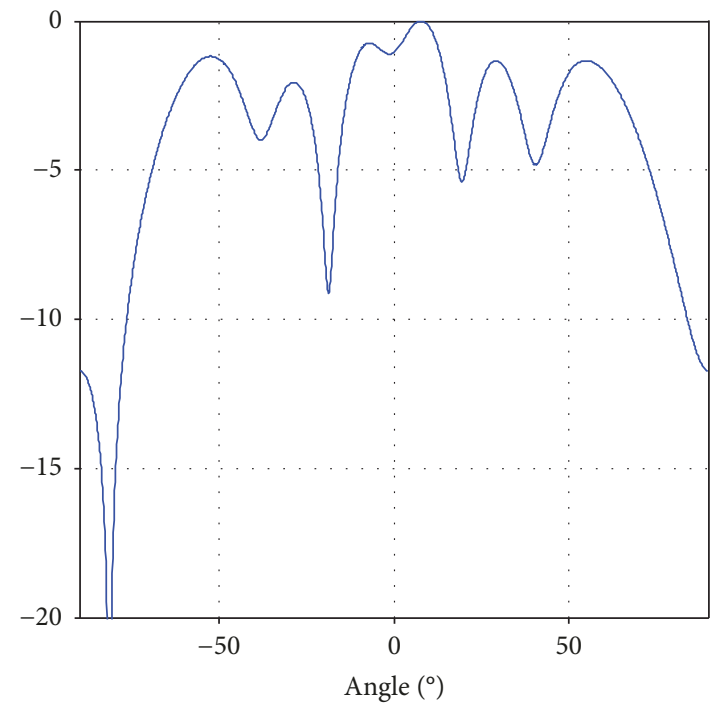

(d)

Figure 3: Normalized beams of phased array: (a) $\theta=0^{\circ}$; (b) $\theta=0^{\circ}, 30^{\circ}$, and $60^{\circ}$; (c) $\theta=0^{\circ}, 3^{\circ}$, and $60^{\circ}$; (d) $\theta=0^{\circ}, 0^{\circ}$, and $60^{\circ}$.

where $\odot$ is the Hadamard product.

$$
\begin{aligned}
\Psi(\theta)= & {\left[1, \exp \left(j \frac{2 \pi\left(f_{0}+\Delta f_{2}\right)}{c} d \sin \theta\right), \cdots,\right.} \\
& \left.\cdot \exp \left(j \frac{2 \pi\left(f_{0}+\Delta f_{M}\right)}{c} d(M-1) \sin \theta\right)\right]^{T}, \\
\Psi(r)= & \exp \left(-j \frac{2 \pi}{c} f_{0} r\right) \cdot \exp \left(-j \frac{2 \pi}{c} \Delta f_{1} r, \cdots,-j \frac{2 \pi}{c} \Delta f_{M} r\right)^{T} .
\end{aligned}
$$

The array factor is

$$
\operatorname{AF}(\theta, r)=\left[\Psi\left(\theta_{1}, r_{1}\right), \cdots, \Psi\left(\theta_{p}, r_{p}\right)\right]
$$

The beam point to $\left(\theta_{1}, r_{1}\right)$ is

$$
P(\theta, r)=w\left(\theta_{1}, r_{1}\right) \cdot \operatorname{AF}(\theta, r)
$$

where $w\left(\theta_{1}, r_{1}\right)=\Psi^{\mathrm{H}}\left(\theta_{1}, r_{1}\right)$.

In order to better illuminate the advantage of FDA in mainlobe jamming suppression, the beampattern of phased array is discussed. Considering the following 4 cases, in case 1 , only echo signal is from $0^{\circ}$ and there is no jamming signal; in case 2 , echo signal and two jamming signals are from $0^{\circ}$, $30^{\circ}$, and $60^{\circ}$, so the two jamming signals are both sidelobe jamming; in case 3 , the echo signal and two jamming signals are from $0^{\circ}, 3^{\circ}$, and $60^{\circ}$, so there is one MLJ and one sidelobe jamming; and in case 4 , the echo and two jamming signals are from $0^{\circ}, 0^{\circ}$, and $60^{\circ}$ and the MLJ has the same direction with real echo signal. The beamforming results are shown in Figure 3. Comparing Figures 3(a) and 3(b), we can get that 


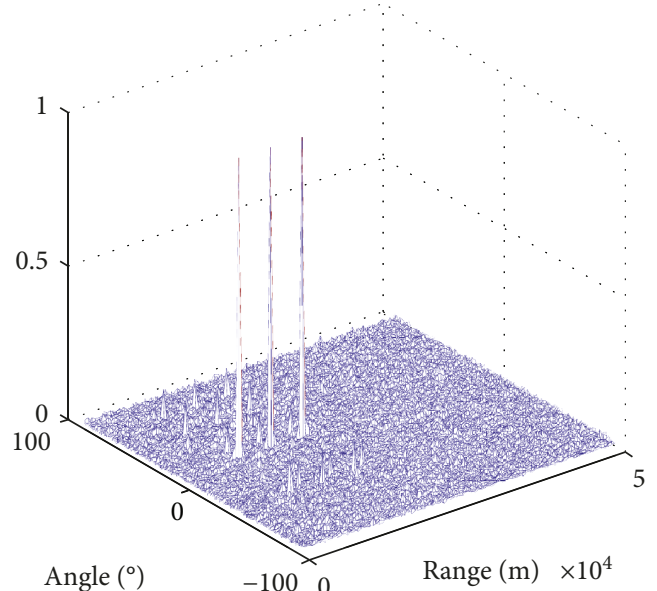

(a)

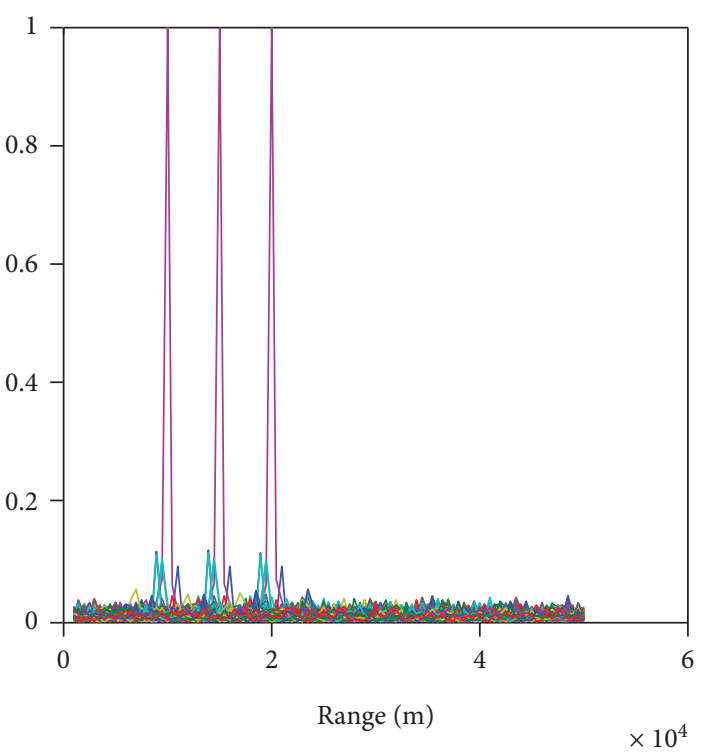

(c)

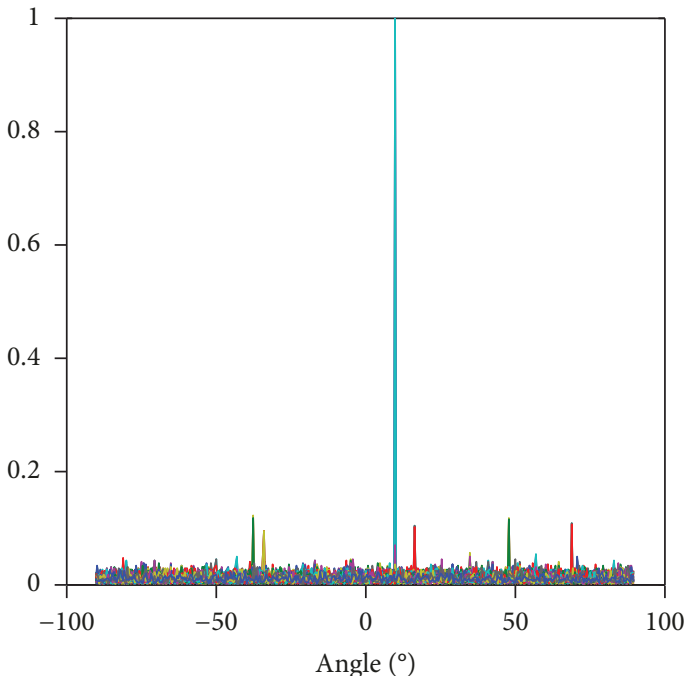

(b)

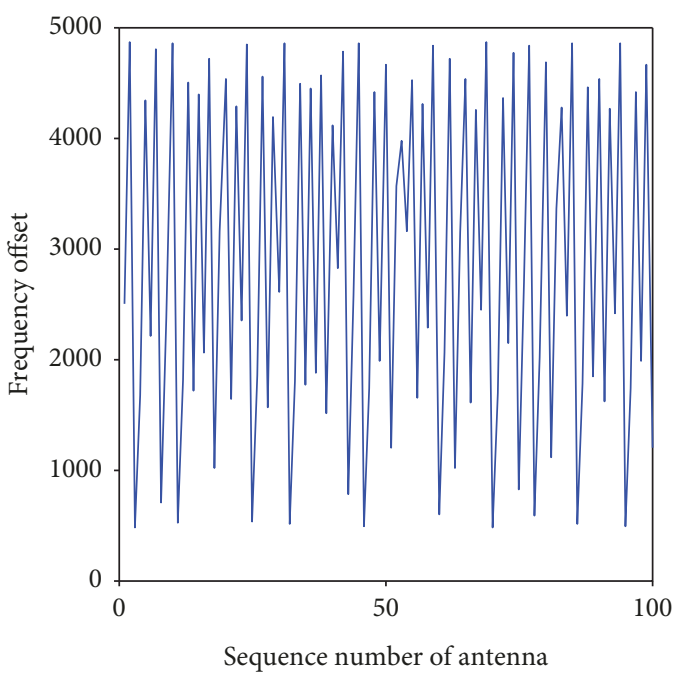

(d)

FIgURE 4: (a) Normalized beams of the proposed FDA; (b) range dimension; (c) angle dimension; (d) frequency offset of different antenna arrays.

the phased array can form nulling in sidelobe jamming direction and real echo signal can be received effectively; from Figure 3(c), we can get that when the phased array is used to suppress MLJ, the mainlobe will drift and sidelobe will raise; from Figure 3(d), we can get that when the jammer and targets are overlapped in the same LOS of radar, the mainlobe is seriously distorted.

MLJ suppression based on the phased array is achieved in the receiver [10-16]. Next, the beamforming of improved FDA proposed by this paper will be discussed to illuminate that radar's performance can also be improved from the transmitter. Assuming there are three targets located at $\left(10^{\circ}, 10 \mathrm{~km}\right),\left(10^{\circ}, 15 \mathrm{~km}\right)$ and $\left(10^{\circ}, 20 \mathrm{~km}\right)$, respectively, the control parameters $\mu=3.9$, initial frequency offset $\Delta f_{1}=5000$
$\mathrm{Hz}, f_{0}=10 \mathrm{GHz}$, and the number of array elements is 100 . Beamforming results of L-FDA are shown in Figure 4.

Comparing Figure 4 to Figure 1, we can see that beams of FDA have been greatly improved. In Figure 4(a), three rangeangle uncoupled mainlobe beams are formed in the desired region and the sidelobe is much lower than the mainlobe, so energy can be effectively focused on the desired area; in Figures $4(\mathrm{~b})$ and $4(\mathrm{c})$, the $3 \mathrm{~dB}$ bandwidth of range and dimension is about $250 \mathrm{~m}$ and $1^{\circ}$, respectively. Frequency offsets of different antenna arrays are shown in Figure 4(d); we can see that the frequency offset floats between 0 and $5 \mathrm{kHz}$; so, by the frequency offset shift method proposed in this paper, this means ideal beampattern can be got through a small frequency offset. 


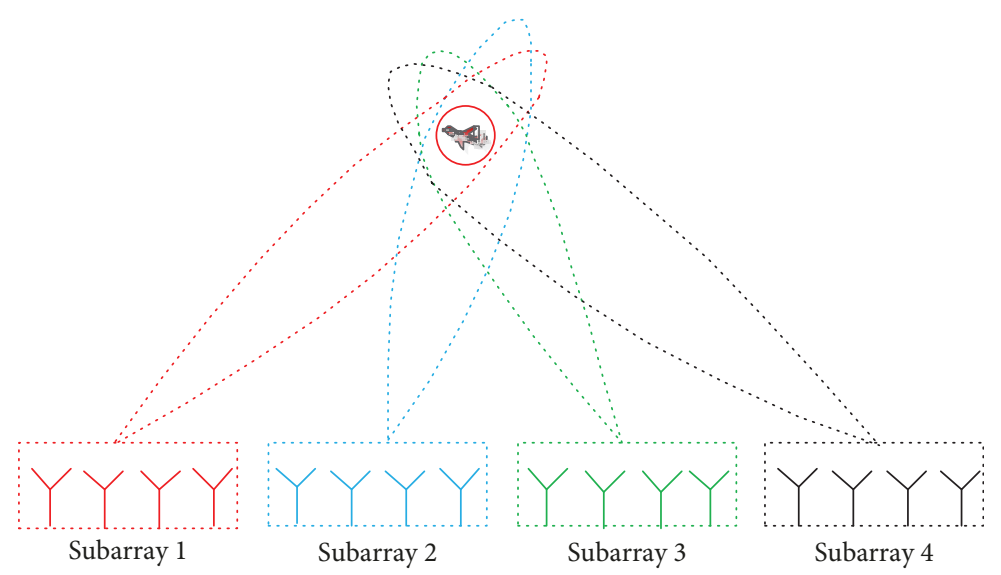

FIgURE 5: Illustration of the FDA-MIMO antenna array $(K=4)$.

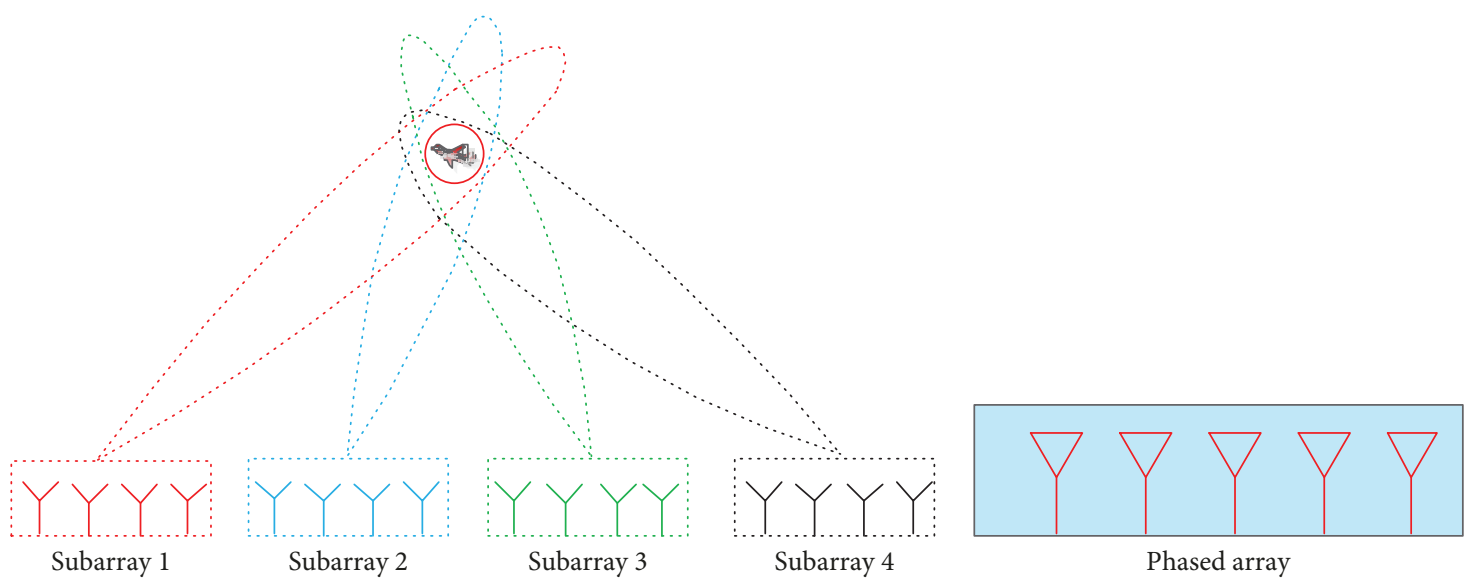

Figure 6: The model of FDA-MIMO.

Compared with the basic FDA and phased array, the proposed FDA based on the logistic map has the following four advantages: (1) since the frequencies of different FDA elements are random, the transmit signal will not be detected accurately and L-FDA has higher anti-jamming ability; (2) because frequencies of elements float around the initial frequency, the difference between the maximum and minimum frequency offsets is much smaller than the basic FDA, which reduces the bandwidth requirement on hardware and cost; (3) the resolution of L-FDA's beam is much better than the basic FDA; and (4) multiple ideal beams will be generated in the direction of different targets, no signal will be transmitted or received in the uninterested area, and this will raise the efficiency of energy utilization.

2.2. FDA-MIMO Radar Based on the Improved FDA. Based on the improved FDA, a new type of FDA-MIMO radar is put forward. Refer to the literature [30], the transmit array of FDA-MIMO consists of $K$ subarrays. All elements of $l$-th subarray are used to coherently emit the signal $\varphi_{l}(t)$, and a beam can be formed toward a certain direction and range, e.g., direction and range of the target, as shown in
Figure 5. For a clearer comparison of FDA-MIMO and phased-MIMO radars, beams of phased-MIMO radars are denoted by dashed lines and beams of FDA-MIMO radars are denoted by solid lines. Different beams can be formed by different subarrays toward different directions or ranges. The data from beams (formed from different subarrays) in the same direction and range contains the information of the same target; thus, these data can be used for coherent processing. And the data from beams in different directions or ranges contain the information of different single target; thus, the information of each target can be obtained simultaneously without signal separation.

The well-separated subarrays are used to form MIMO radar. Different waveforms transmitted by different subarrays should be orthogonal. In this paper, the receiver is a phased array, which is made up of $N$ antenna elements. In each FDA-MIMO radar, the signals transmitted from subarrays which formed toward the same direction and distance are used to synthesize a signal toward the target. Therefore, for one target, each FDA-MIMO radar will transmit a signal to observe it and each FDA-MIMO radar can be treated as one transmitter. 


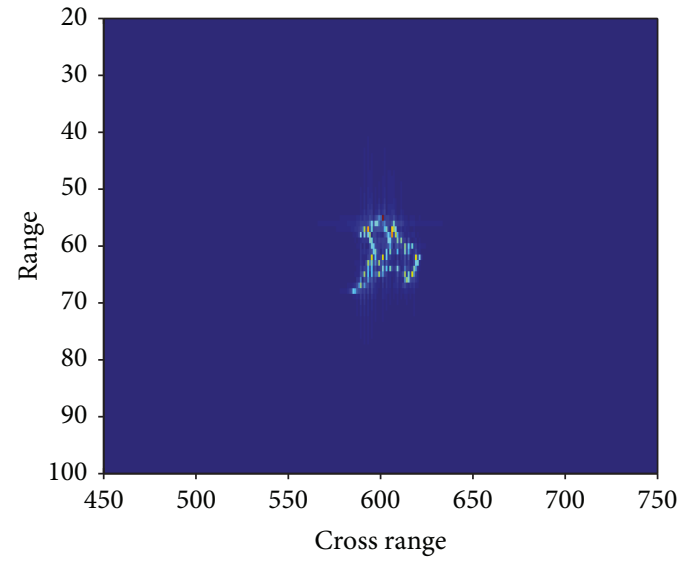

(a)

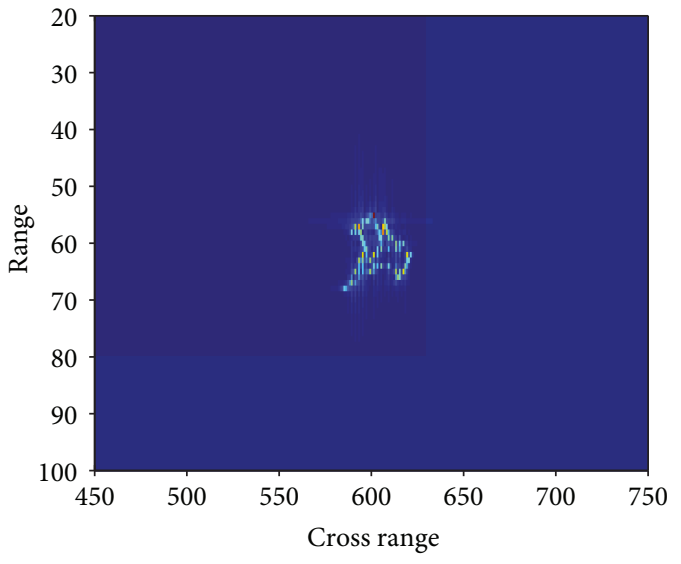

(b)

FIGURE 7: (a) ISAR image with LFM signal. (b) ISAR image with SFCS signal.

We combine the advantages of FDA and MIMO radar in range-angle-uncoupled beampattern: coherent array gain, waveform diversity, better beamsteering performance, and high SNR gain; FDA-MIMO radar is applied to transmit different signals to jammers and targets, respectively. We consider the co-located MIMO radar as showed in Figure 6 $(K=4, N=5)$. In Figure 6 , the elements in each subarray emits signals with the same envelop and the subarray is worked in FDA mode.

2.3. Imaging Based on the FDA-MIMO Radar. The signal emitted by the $l$-th subarray is

$$
s_{l}(t)=\varphi_{l}(t) \exp \left(j 2 \pi f_{l} t\right), \quad 0 \leq t \leq T,
$$

where $\varphi_{l}(t)$ is the signal's envelop of the $l$-th subarray, $f_{l}$ is the carrier frequency of $l$-th subarray, and $T$ is the pulse duration. The signals transmitted by different subarrays satisfy

$$
\int_{T} s_{l}(t) s_{p}^{*}(t-\tau)=0, \quad l \neq p, \forall \tau,
$$

where $\tau$ is time delay and the superscript $*$ denotes a conjugate operator.

The signals emitted by different subarrays are orthogonal or irrelevant. Considering the far-field situation, when signal is emitted by the $m$-th element of $l$-th subarray and received by the $h$-th element of receiving array, the round trip time delay is

$$
\begin{aligned}
\tau_{l, m, h}^{\prime} & =\tau_{0}+\tau_{l, m, h} \\
& =\frac{R_{t l}}{c}+\frac{R_{r}}{c}-\frac{d_{1}(m-1) \sin \theta_{1}+d_{2}(h-1) \sin \theta_{2}}{c},
\end{aligned}
$$

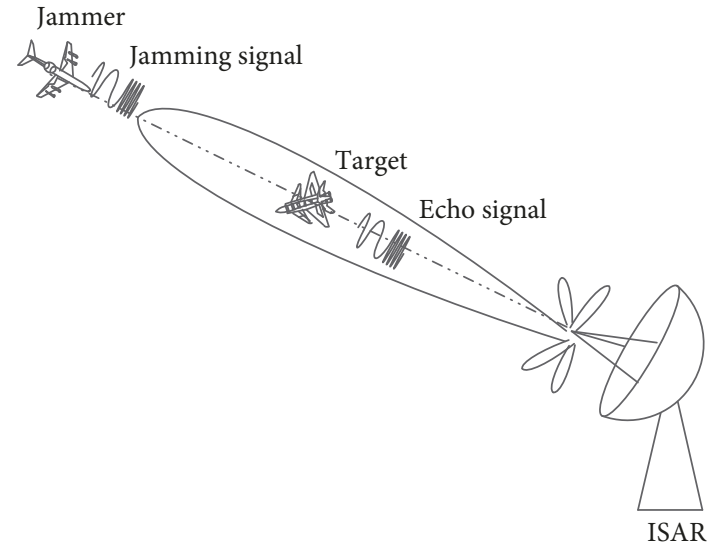

Figure 8: The geometric relation diagram of MLJ.

where $R_{t l}$ and $R_{r}$ are distances from the target to the first element of $l$-th subarray and receiving array, respectively, $d_{1}$ and $d_{2}$ is the element space of subarrays and phased array, respectively, $\theta_{1}$ and $\theta_{2}$ is the angle of FDAMIMO and phased array, respectively, $\tau_{0}=\left(R_{t l}+R_{r}\right) / c$ is the common time delay, and $\tau_{l, m, h}$ is the time delay between elements [21].

The receiving signal corresponding to $\tau_{l, m, h}{ }^{\prime}$ is

$$
s r_{l, m, h}(t)=\varphi_{l}\left(t-\tau_{l, m, h}^{\prime}\right) \cdot \exp \left(j 2 \pi f_{l}\left(t-\tau_{l, m, h}^{\prime}\right)\right) .
$$

The receiving snapshot of FDA-MIMO radar can be expressed as a vector:

$$
x_{s}=\left[y_{1,1,1}, y_{1,1,2}, \cdots, y_{1,1, N}, \cdots, y_{K, M, N}\right]^{T}=\xi b\left(\theta_{2}\right) \otimes a\left(\theta_{1}, r\right) \text {, }
$$




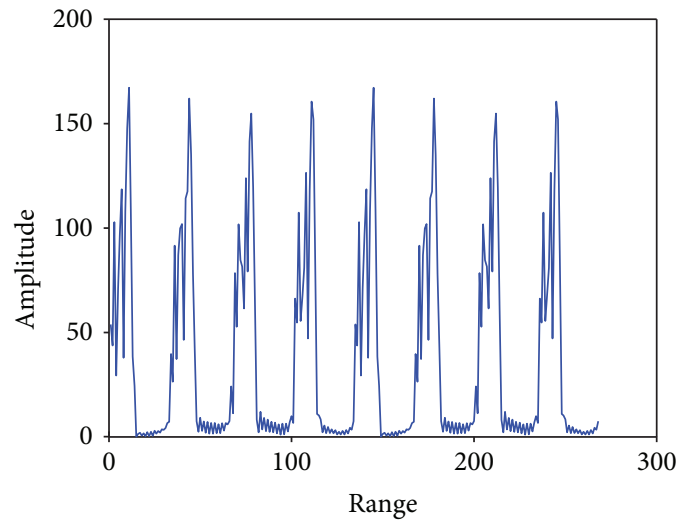

(a)

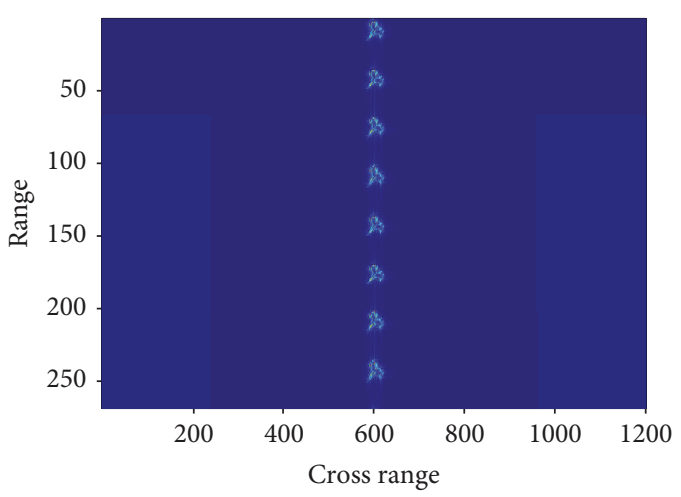

(b)

FIGURE 9: Sub-Nyquist sampling jamming: (a) the HRRP of Sub-Nyquist sampling jamming; (b) the ISAR image of Sub-Nyquist sampling jamming.

where $x_{s} \in C^{K M N \times 1}$, the superscript $T$ denotes the operation of transpose, $a\left(\theta_{1}, r\right) \in C^{K M \times 1}$, and $b\left(\theta_{2}\right) \in C^{N \times 1}$. The expression of $a\left(\theta_{1}, r\right)$ and $b\left(\theta_{2}\right)$ is

$$
\begin{aligned}
& a\left(\theta_{1}, r\right)=\exp \left(j 2 \pi \frac{f_{0}\left(R_{t l}+R_{r}\right)}{c}\right) \\
& \cdot\left[1, \exp \left(-j 4 \pi \frac{\Delta f}{c}(m-1)\left(R_{t l}+R_{r}\right)\right), \cdots,\right. \\
& \left.\cdot \exp \left(-j 4 \pi \frac{\Delta f}{c}(M-1)\left(R_{t l}+R_{r}\right)\right)\right]^{T} \\
& \odot\left[1, \exp \left(j 2 \pi \frac{f_{l}}{c} d_{1}(m-1) \sin \left(\theta_{1}\right)\right), \cdots,\right. \\
& \left.\cdot \exp \left(j 2 \pi \frac{f_{l}}{c} d_{1}(M-1) \sin \left(\theta_{1}\right)\right)\right]^{T} \\
& \approx \exp \left(j 2 \pi \frac{f_{0}\left(R_{t l}+R_{r}\right)}{c}\right) \\
& \cdot\left[1, \exp \left(-j 4 \pi \frac{\Delta f}{c}(m-1)\left(R_{t l}+R_{r}\right)\right), \cdots,\right. \\
& \left.\exp \left(-j 4 \pi \frac{\Delta f}{c}(M-1)\left(R_{t l}+R_{r}\right)\right)\right]^{T} \\
& \odot\left[1, \exp \left(j 2 \pi \frac{d_{1}}{\lambda_{0}}(m-1) \sin \left(\theta_{1}\right)\right), \cdots,\right. \\
& \left.\cdot \exp \left(j 2 \pi \frac{d_{1}}{\lambda_{0}}(M-1) \sin \left(\theta_{1}\right)\right)\right]^{T}, \\
& b\left(\theta_{2}\right)=\left[1, \exp \left(j 2 \pi \frac{d_{2}}{\lambda_{0}} \sin \theta_{2}\right), \cdots,\right. \\
& \left.\cdot \exp \left(j 2 \pi(N-1) \frac{d_{2}}{\lambda_{0}} \sin \theta_{2}\right)\right]^{T} \text {, }
\end{aligned}
$$

where $\lambda_{0}$ is the wavelength corresponding to the reference carrier frequency $f_{0}$.

Due to the small frequency offset, there are some differences between signals emitted by FDA and phased array, and the imaging results based on FDA need to be further
TABLE 1: The chirp rates of signals transmitted to the jammer and target.

\begin{tabular}{lcc}
\hline Chirp rate $(\mathrm{Hz} / \mathrm{s})$ & Jammer & Target \\
\hline LFM & $3 \times 10^{13} / 6 \times 10^{12}$ & $3 \times 10^{14}$ \\
SFCS & $3 \times 10^{13} / 6 \times 10^{12}$ & \\
\hline
\end{tabular}

analyzed. Because LFM and SFCS signals are widely applied in ISAR imaging, the ISAR image of these two signals based on the FDA radar will be discussed, respectively, in the following.

First, considering that the FDA-MIMO radar emits LFM signal, the signal emitted by the $m$-th element of $l$-th subarray is

$$
\begin{aligned}
s_{l, m}\left(\widehat{t}, t_{m}\right)= & \sigma \operatorname{rect}\left(\frac{\widehat{t}}{T_{p}}\right) \cdot \exp \left(j 2 \pi\left(f_{0}+(m-1) \Delta f\right) t\right. \\
& \left.+\frac{1}{2} \gamma t^{2}\right), \quad|\widehat{t}| \leq \frac{T_{p}}{2}, m=1,2, \ldots, M,
\end{aligned}
$$

where $T_{p}$ is time width. So, signal emitted by $l$-th subarray can be expressed by

$$
\begin{aligned}
s_{l}\left(\widehat{t}, t_{m}\right)= & \sigma \operatorname{rect}\left(\frac{\widehat{t}}{T_{p}}\right) \cdot \sum_{m=1}^{M} \exp \left(j 2 \pi\left(f_{0}+(m-1) \Delta f\right) t\right. \\
& \left.+\frac{1}{2} \gamma \widehat{t}^{2}\right), \quad|\widehat{t}| \leq \frac{T_{p}}{2} .
\end{aligned}
$$

The reference signal of $l$-th subarray is

$$
\begin{aligned}
s_{l, \mathrm{ref}}(t)= & \operatorname{rect}\left(\left(\widehat{t}-\left(2 R_{\mathrm{ref}} / c\right)\right) / T_{\mathrm{ref}}\right) \cdot \exp \left(j 2 \pi f_{l}\left(t-\frac{R_{\mathrm{ref}}}{c}\right)\right. \\
& \left.+\frac{1}{2} \gamma\left(\hat{t}-\frac{R_{\mathrm{ref}}}{c}\right)^{2}\right), \quad|\widehat{t}| \leq \frac{T_{p}}{2},
\end{aligned}
$$

where $R_{\text {ref }}$ is the distance between the target and $l$-th subarray and $T_{\text {ref }}$ is the time width of reference signal. 


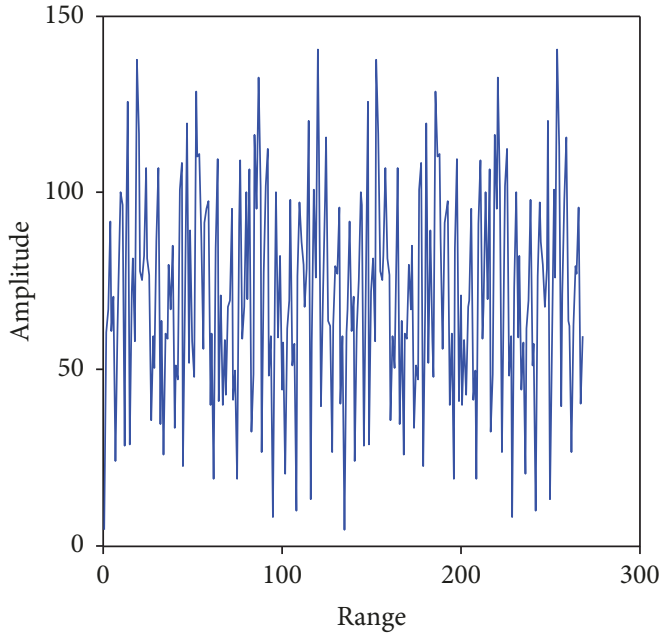

(a)

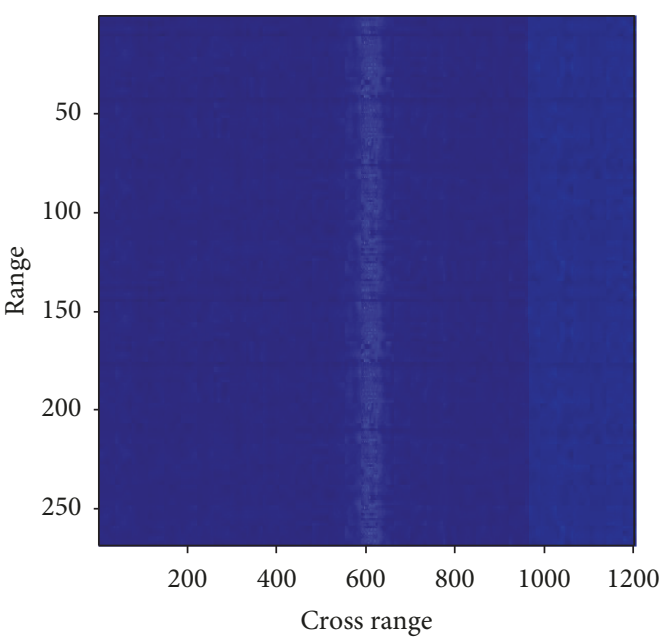

(c)

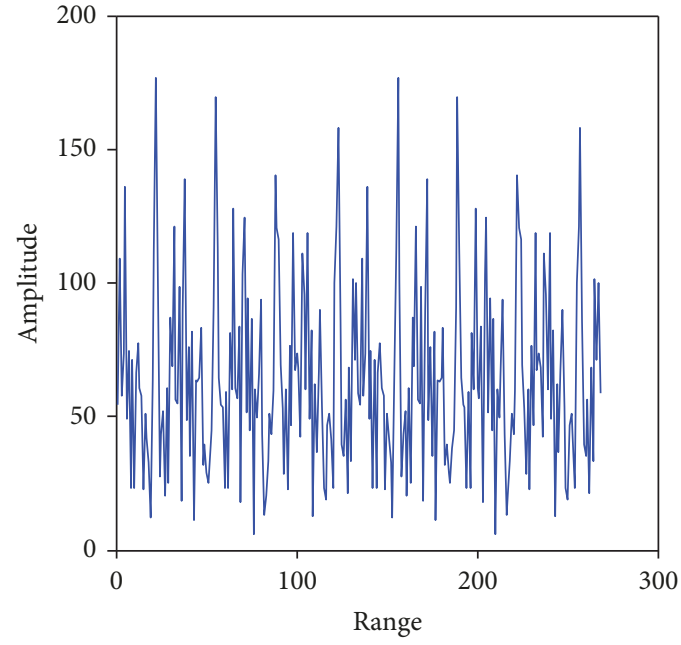

(b)

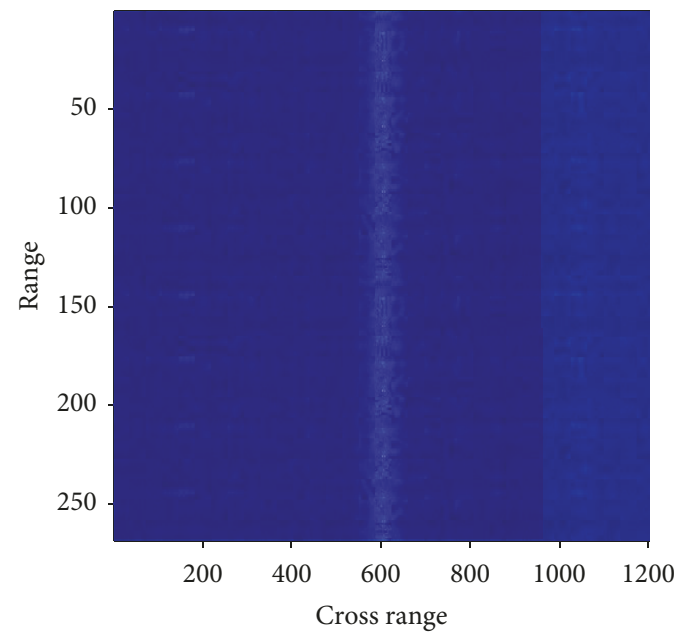

(d)

FIGURE 10: Anti-jamming effect. (a) HRRP when the jamming chirp rate is one-tenth of the target echo; (b) HRRP when the jamming chirp rate is fiftieth of the target echo; (c) ISAR image of jamming signal when the chirp rate is one-tenth of the target echo; (d) ISAR image of jamming signal when the chirp rate is fiftieth of the target echo.

Then, considering the FDA-MIMO radar emits SFCS signal, the $i$-th subpulse emitted by $m$-th element of $l$-th subarray is

$$
\begin{aligned}
s_{l, m}(\widehat{t}, i)= & \sigma \operatorname{rect}\left(\frac{\hat{t}-i T_{r}}{T_{1}}\right) \\
& \cdot \exp \left(j 2 \pi \left(f_{0}+i \delta_{f}\right.\right. \\
& \left.+(m-1) \Delta f)\left(\widehat{t}-i T_{r}\right)+j \theta_{i}\right) \\
& \cdot \exp \left(j \pi \gamma\left(\widehat{t}-i T_{r}\right)^{2}\right), \\
|\widehat{t}| \leq & \frac{T_{1}}{2}, m=1,2, \ldots, M, i=0,1, \ldots, N-1,
\end{aligned}
$$

where $\delta_{f}$ is the stepped frequency of SFCS signal, $T_{r}$ is the repetition interval of subpulses, $T_{1}$ is the time width of subpulse, $\gamma$ is the chirp rate of subpulse, and $\theta_{i}$ is the initial phase of $i$-th subpulse.
The SFCS signal emitted by $l$-th subarray can be expressed as

$$
\begin{aligned}
s_{l}(\hat{t}, i)= & \sigma \sum_{i=0}^{N-1} \sum_{m=1}^{M} \operatorname{rect}\left(\frac{\hat{t}-i T_{r}}{T_{1}}\right) \cdot \exp \left(j 2 \pi \left(f_{0}+i \delta_{f}\right.\right. \\
& \left.+(m-1) \Delta f)\left(\widehat{t}-i T_{r}\right)+j \theta_{i}\right) \\
& \cdot \exp \left(j \pi \gamma\left(t \wedge-i T_{r}\right)^{2}\right), \quad|\widehat{t}| \leq \frac{T_{1}}{2} .
\end{aligned}
$$

For $l$-th subarray, assumed the first element's signal as reference. The simulation parameters of L-FDA are identical with Section 2. The carrier frequency of ISAR is $10 \mathrm{GHz}$, and bandwidth is $300 \mathrm{MHz}$.

Figures $7(\mathrm{a})$ and $7(\mathrm{~b})$ are images of FDA-MIMO radar when it emits LFM and SFCS signals, respectively. We can get that for L-FDA, the ISAR image can also be obtained as same as phased array, so FDA is feasible to ISAR imaging. 


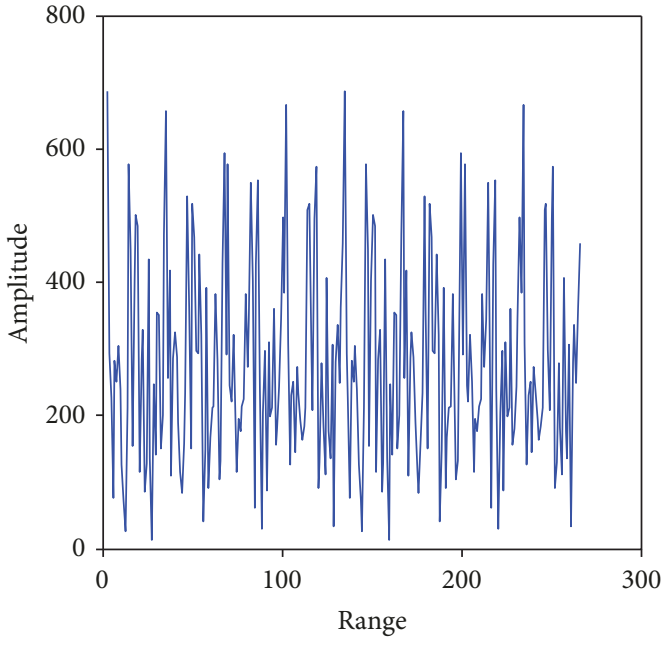

(a)

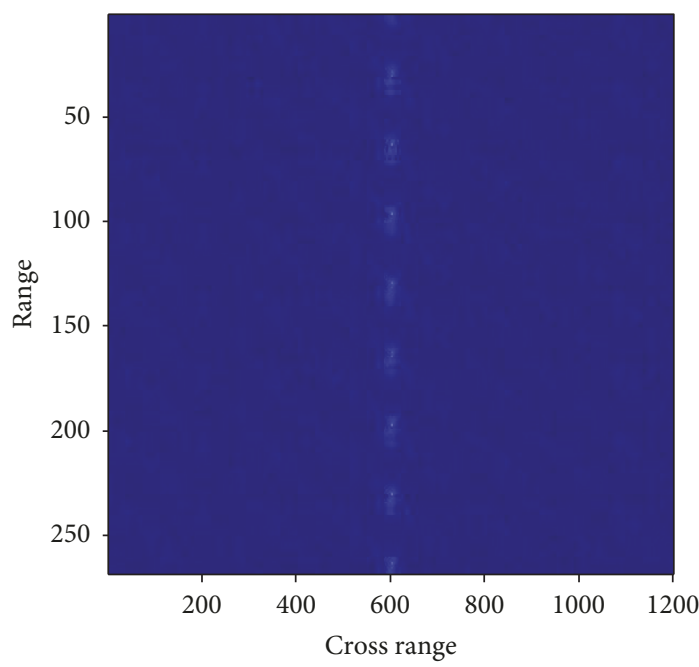

(c)

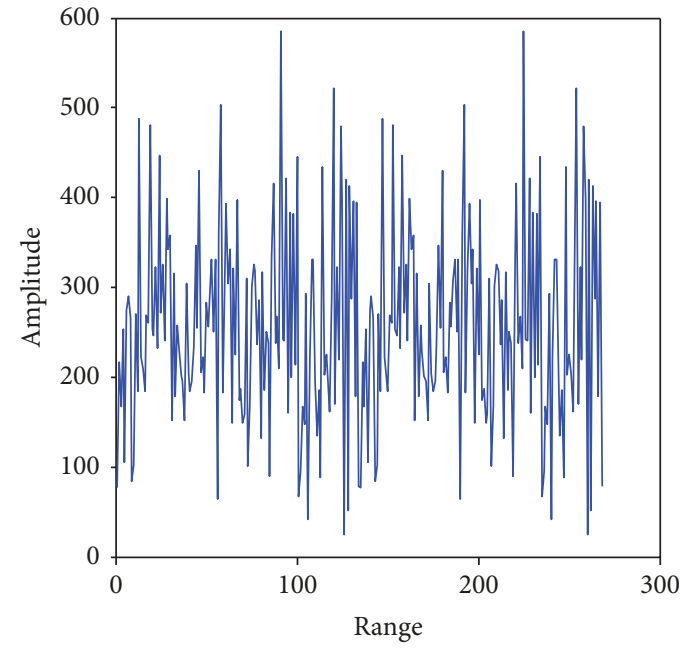

(b)

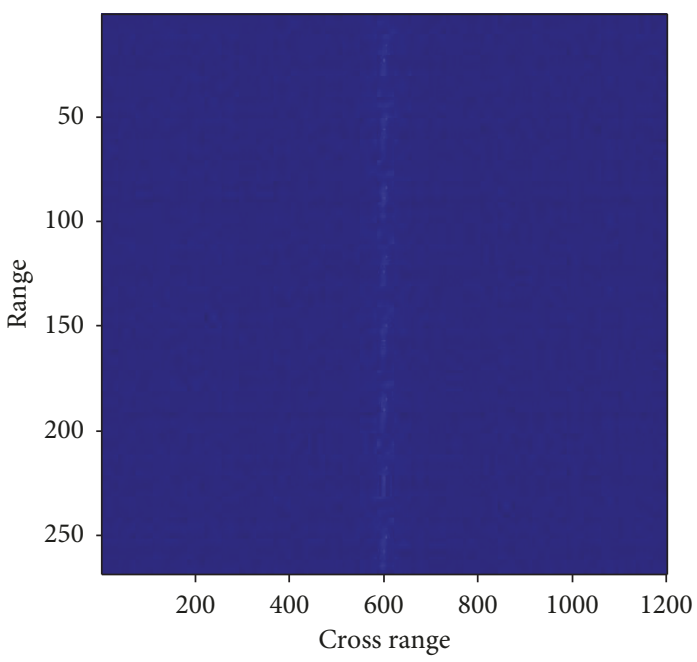

(d)

FIGURE 11: Anti-jamming effect. (a) HRRP when the jamming chirp rate is one-tenth of the target echo; (b) HRRP when the jamming chirp rate is fiftieth of the target echo; (c) ISAR image of jamming signal when the chirp rate is one-tenth of the target echo; (d) ISAR image of jamming signal when the chirp rate is fiftieth of the target echo.

\section{Experiments}

In order to suppress the MLJ, we will emit different signals to the target and jammer by the proposed FDA-MIMO radar in the following. Because sub-Nyquist sampling jamming has attracted much attention, the MLJ suppression effect is examined by sub-Nyquist sampling jamming. And detailed principle of sub-Nyquist sampling jamming is explained in reference [1].

The geometric relation between targets, jammer, and FDA-MIMO radar is shown in Figure 8; range and angle parameters of the targets and jammer can be obtained by the method proposed in the literatures [21, 31]. The target echoes and jamming signal can be discriminated by the method proposed in [32]. In order to make full use of range-angle-dependent beampattern of the improved FDA, we consider transmitting different signals to the jammer and targets based on the pulse diversity theory. The signals are altered in two aspects: (1) different waveforms (LFM or SFCS) are applied in the anti-jamming method and (2) chirp rates of signals are jittered.

Following simulation results are utilized to validate the effectiveness of the proposed anti-jamming method. First, the sub-Nyquist sampling jamming is presented when signal is emitted by FDA-MIMO radar. Assumed the X-band radar is operated at $10 \mathrm{GHz}$, and bandwidth is $300 \mathrm{MHz}$. A total of 108 pulses are transmitted within a coherent image integration time of $1.1268 \times 10^{-6} \mathrm{~s}$, and the sampling interval of sub-Nyquist is $T_{s}=8.3776 \times 10^{-8} \mathrm{~s}$; multiple deceptive images are induced in Figure 9.

Assumed the FDA-MIMO system is constructed with $M r=2$ transmitters, and the antenna number of receiving phased array is $N=100$. Each transmitter is a FDAMIMO radar with 100 elements. There is one target and one jammer located at $\left(10^{\circ}, 200 \mathrm{~km}\right)$ and $\left(10^{\circ}, 205 \mathrm{~km}\right)$ as showed in Figure 8 . Each transmitter is divided into $K=4$ 
subarrays with the fully overlapped method. The first two subarrays are toward the jammer, and last two subarrays are toward the target.

In simulation, the carrier frequencies of signals transmitted to the target and jammer are $10 \mathrm{GHz}$ and $11 \mathrm{GHz}$, respectively; the chirp rates of different waveforms are shown in Table 1.

Firstly, we just consider chirp rate jitter, which means ISAR emits LFM signal to both the jammer and the target. Figures 10(a) and 10(c) are the HRRP and ISAR images when the chirp rate of jamming signal is taken as $3 \times 10^{13} \mathrm{~Hz} / \mathrm{s}$ (the chirp rate of jamming signal is one-tenth of the target echoes); Figures 10(b) and 10(d) are the HRRP and ISAR images when the chirp rate of jamming signal is $6 \times 10^{12} \mathrm{~Hz} / \mathrm{s}$ (the chirp rate of jamming signal is fiftieth of the target echoes). Compared with the jamming image in Figure 9, the HRRP and the ISAR image of jamming signal is blurred and the false targets cannot be formed successfully. But in the radar work area, the jamming signal can also cause occlusion; this will affect the target recognition. This means just considering the chirp rate jitter cannot get well anti-jamming effect. In the following, besides chirp rate jitter, waveform diversity is also considered in jamming suppression.

In the following, we consider the waveform changing and chirp rate jitter at the same time, Figure 11 shows the antijamming result when LFM signal transmits to the target and SFCS signal transmits to the jammer. Figures 11(a) and 11(b) are HRRP; Figures 11(c) and 11(d) are ISAR images. Compared to Figure 10, jamming is eliminated more thoroughly. It means by combining the waveform changing and chirp rate jitter, jamming can be better suppressed compared with chirp rate jitter only.

\section{Conclusion}

In this paper, FDA-MIMO radar is applied to suppress MLJ. In order to overcome the periodicity and rangeangle coupling of FDA's beampattern, an improved FDA model based on the logistic map is proposed. Then, the FDA-MIMO based on the proposed FDA is introduced, and an anti-jamming method is proposed in this paper. With the advantage of FDA-MIMO radar, by transmitting different signals to the target and jammer, the well MLJ suppression effect will be obtained easily without complex algorithms. The simulation results demonstrate the effectiveness of the anti-jamming method.

Some in-depth work will be studied in the future on the following points:

(1) Because the radar resource is limited, the resource saturation will be an important problem when there are too many targets in the radar work area. In this case, the available subarrays and limited radar power will be not enough to be allocated for every target. Thus, reasonable and effective resource scheduling algorithms are important for exploiting the benefits of FDA-MIMO radar

(2) In this paper, we just consider the LFM and SFCS signals to MLJ suppression. Waveform design has been an effective measure for anti-jamming. If the signals transmitted to the jammer and the target are well designed, jamming can be eliminated better. Thus, designing waveforms with well self-correlation and cross-correlation features is very important for the mainlobe jamming suppression with FDA-MIMO

\section{Data Availability}

The data used to support the findings of this study are available from the corresponding author upon request.

\section{Conflicts of Interest}

The authors declare that there is no conflict of interest regarding the publication of this paper.

\section{Acknowledgments}

This work was supported in part by the National Natural Science Foundation of China under Grant 61631019, Young Scientists Fund of the National Natural Science Foundation of China under Grant 61703412, and Natural Science Foundation Research Program of Shaanxi Province under Grant 2018JM6072.

\section{References}

[1] X. Pan, W. Wang, D. Feng, Y. Liu, Q. Fu, and G. Wang, "On deception jamming for countering bistatic ISAR based on sub-Nyquist sampling," IET Radar, Sonar \& Navigation, vol. 8, no. 3, pp. 173-179, 2014.

[2] W. Wang, X.-Y. Pan, Y.-C. Liu, D.-J. Feng, and Q.-X. Fu, "SubNyquist sampling jamming against ISAR with compressive sensing," IEEE Sensors Journal, vol. 14, no. 9, pp. 3131-3136, 2014.

[3] X. Pan, J. Liu, J. Chen, Q. Xie, and X. Ai, "Sub-Nyquist sampling jamming against chirp-ISAR with CS-D range compression," IEEE Sensors Journal, vol. 18, no. 3, pp. 1140-1149, 2018.

[4] X.-Y. Pan, W. Wang, and G.-Y. Wang, "Sub-Nyquist sampling jamming against chirp-ISAR with CS-based HRRP reconstruction," IEEE Sensors Journal, vol. 16, no. 6, pp. 15971602, 2018.

[5] Y. Li, Y. Wu, C. Yin, and Z. Wang, "Planar array ISAR imaging and anti-jamming performance analyzation," in Proceedings of 2011 IEEE CIE International Conference on Radar, pp. 15311534, Chengdu, China, October 2011.

[6] G. Li, H. Zhang, X. Wang, and X. G. Xia, "ISAR 2-D imaging of uniformly rotating targets via matching pursuit," IEEE Transactions on Aerospace and Electronic Systems, vol. 48, no. 2, pp. 1838-1846, 2012.

[7] L. Ding, W. Chen, W. Zhang, and H. V. Poor, "MIMO radar imaging with imperfect carrier synchronization: a point spread function analysis," IEEE Transactions on Aerospace and Electronic Systems, vol. 51, no. 3, pp. 2236-2247, 2015.

[8] K.-B. Yu and D. J. Murrow, "Adaptive digital beamforming for angle estimation in jamming," IEEE Transactions on Aerospace and Electronic Systems, vol. 37, no. 2, pp. 508-523, 2001.

[9] S. H. Moon, D. S. Han, H. S. Oh, and M. J. Cho, "Monopulse angle estimation with constrained adaptive beamforming 
using simple mainlobe maintenance technique," in IEEE Military Communications Conference, 2003. MILCOM 2003, pp. 1365-1373, Boston, MA, USA, October 2003.

[10] S. Applebaum and D. Chapman, "Adaptive arrays with main beam constraints," IEEE Transactions on Antennas and Propagation, vol. 24, no. 5, pp. 650-662, 1976.

[11] U. Nickel, "Monopulse estimation with adaptive arrays," IEE Proceedings F - Radar and Signal Processing, vol. 140, no. 5, pp. 303-308, 1993.

[12] S.-J. Yu and J.-H. Lee, "Efficient eigenspace-based array signal processing using multiple shift-invariant subarrays," IEEE Transactions on Antennas and Propagation, vol. 47, no. 1, pp. 186-194, 2002.

[13] R. Li, Y. Wang, and S. Wan, "Research of reshaping adapted pattern under mainlobe interference conditions," Modern Radar, vol. 24, no. 3, pp. 50-53, 2002.

[14] X. Yang, Z.Zhang, T.Zeng, T. Long, and T. K. Sarkar, "Mainlobe interference suppression based on eigen-projection processing and covariance matrix reconstruction," IEEE Antennas and Wireless Propagation Letters, vol. 13, pp. 1369-1372, 2014.

[15] J. Yang and C. Liu, "Improved mainlobe interference suppression based on blocking matrix preprocess," Journal of Electrical and Computer Engineering, vol. 2015, Article ID 964901, 8 pages, 2015.

[16] J. Qian and Z. He, "Mainlobe interference suppression with eigenprojection algorithm and similarity constraints," Electronics Letters, vol. 52, no. 3, pp. 228-230, 2016.

[17] P. Antonik, M. C. Wicks, H. D. Griffiths, and C. J. Baker, "Frequency diverse array radars," in 2006 IEEE Conference on Radar, pp. 215-217, Verona, NY, USA, April 2006.

[18] W. Khan, I. M. Qureshi, and S. Saeed, "Frequency diverse array radar with logarithmically increasing frequency offset," IEEE Antennas and Wireless Propagation Letters, vol. 14, pp. 499502, 2015.

[19] Y. Wang, G. Huang, and W. Li, "Transmit beampattern design in range and angle domains for MIMO frequency diverse array radar," IEEE Antennas and Wireless Propagation Letters, vol. 16, pp. 1003-1006, 2017.

[20] W. Q. Wang, "Phased-MIMO radar with frequency diversity for range-dependent beamforming," IEEE Sensors Journal, vol. 13, no. 4, pp. 1320-1328, 2015.

[21] J. Xu, G. Liao, S. Zhu, L. Huang, and H. C. So, "Joint range and angle estimation using MIMO radar with frequency diverse array," IEEE Transcation on Signal Processing, vol. 63, no. 13, pp. 3396-3410, 2015.

[22] J. Xu, S. Zhu, and G. Liao, "Space-time-range adaptive processing for airborne radar systems," IEEE Sensors Journal, vol. 15, no. 3, pp. 1602-1610, 2015.

[23] A. Abdalla, W. Q. Wang, Y. Zhao, S. Mohamed, and B. Tang, "Subarray-based FDA radar to counteract deceptive ECM signals," EURASIP Journal on Advances in signal Processing, vol. 58, no. 6, 3151 pages, 2010.

[24] X. Jingwei, J. Kang, and G. Liao, "Mainlobe deceptive jammer suppression with FDA-MIMO radar," in 2018 IEEE 10th Sensor Array and Multichannel Signal Processing Workshop (SAM), pp. 504-508, Sheffield, UK, July 2018.

[25] J. Xu, S. Zhu, and G. Liao, "Range ambiguous clutter suppression for airborne FDA-STAP radar," IEEE Journal of Selected Topics in Signal Processing, vol. 9, no. 8, pp. 1620-1631, 2015.
[26] X. Jingwei, Z. Shengqi, L. Guisheng, and Z. Yuhong, "An overview of frequency diverse array radar technology," Journal of Radars, vol. 7, no. 2, pp. 167-182, 2018.

[27] W.-Q. Wang, H. C. So, and H. Shao, "Nonuniform frequency diverse array for range-angle imaging of targets," IEEE Sensors Journal, vol. 14, no. 8, pp. 2469-2476, 2014.

[28] M. Andrecut, "Logistic map as a random number generator," International Journal of Modern Physics B, vol. 12, no. 9, pp. 921-930, 1998.

[29] Y. Liu, H. Ruan, L. Wang, and A. Nehorai, "The random frequency diverse array: a new antenna structure for uncoupled direction-range indication in active sensing," IEEE Journal of Selected Topics in Signal Processing, vol. 11, no. 2, pp. 295308, 2017.

[30] Y.-J. Chen, Q. Zhang, Y. Luo, and K.-M. Li, "Multi-target radar imaging based on phased-MIMO technique-part I: imaging algorithm," IEEE Sensors Journal, vol. 17, no. 19, pp. 61856197, 2017.

[31] W.-Q. Wang and H. Shao, "Range-angle localization of targets by a double-pulse frequency diverse array radar," IEEE Journal of Selected Topics in Signal Processing, vol. 8, no. 1, pp. 106114, 2014.

[32] J. Xu, G. Liao, S. Zhu, and H. C. So, "Deceptive jamming suppression with frequency diverse MIMO radar," Signal Processing, vol. 113, pp. 9-17, 2015. 


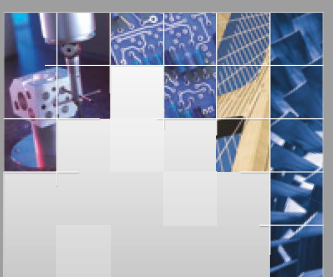

\section{Enfincering}
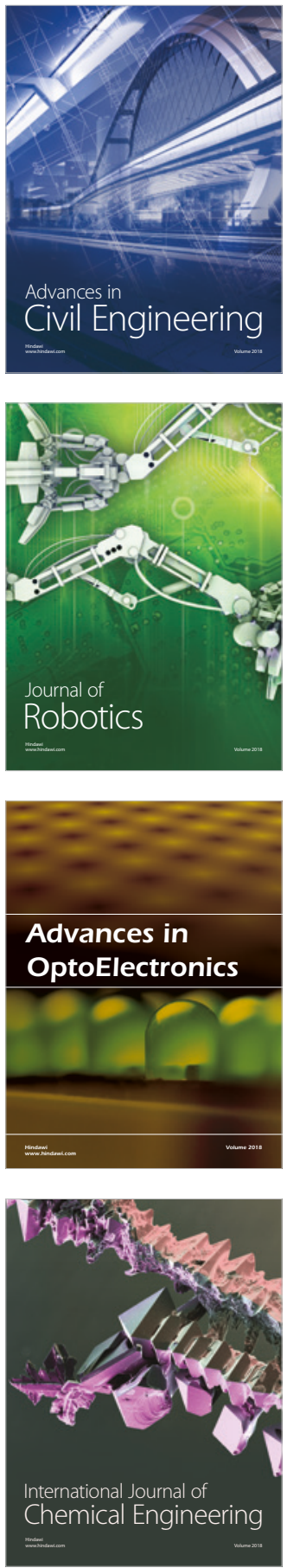

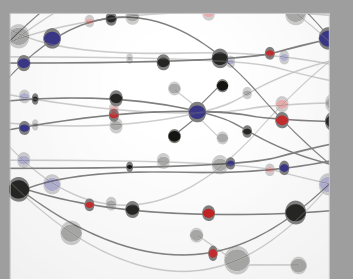

\section{Rotating \\ Machinery}

The Scientific World Journal

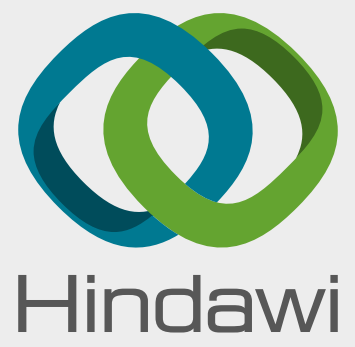

Submit your manuscripts at

www.hindawi.com
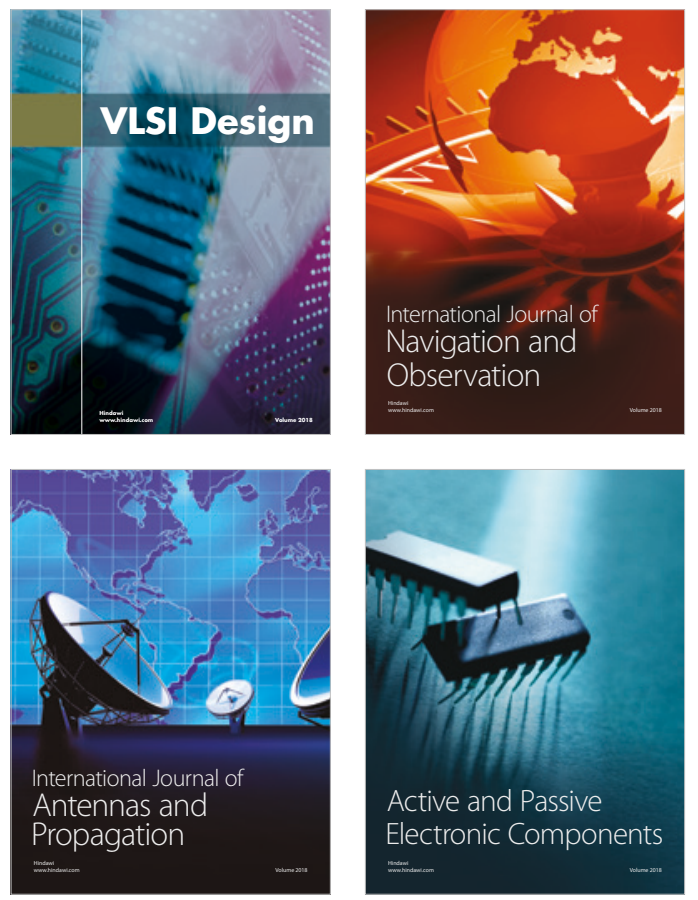
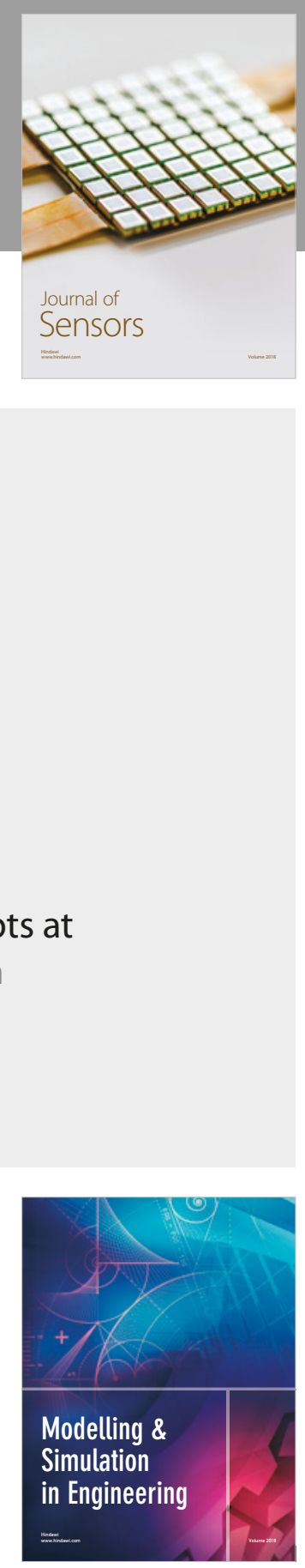

\section{Advances \\ Multimedia}
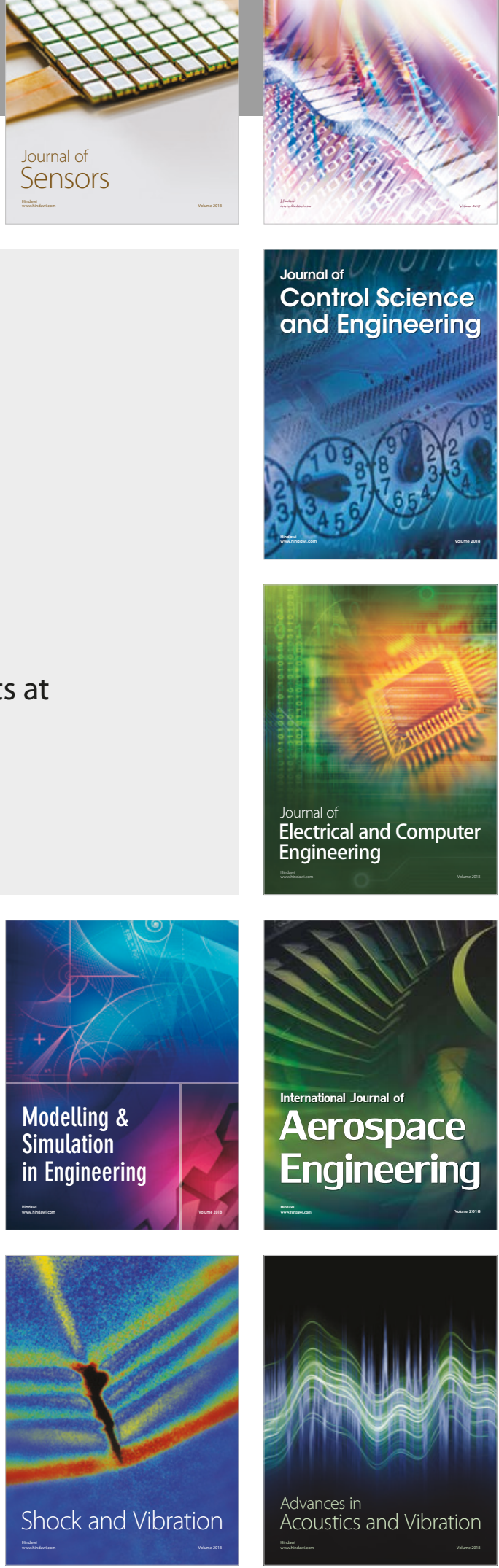